HUTP-99/A055

hep-th/9911001

\title{
Superconformal Multi-Black Hole Moduli Spaces in Four Dimensions
}

\author{
Alexander Maloney, Marcus Spradlin and Andrew Strominger \\ Department of Physics \\ Harvard University \\ Cambridge, MA 02138
}

\begin{abstract}
Quantum mechanics on the moduli space of $N$ supersymmetric Reissner-Nordstrom black holes is shown to admit 4 supersymmetries using an unconventional supermultiplet which contains $3 N$ bosons and $4 N$ fermions. A near-horizon limit is found in which the quantum mechanics of widely separated black holes decouples from that of stronglyinteracting, near-coincident black holes. This near-horizon theory is shown to have an enhanced $D(2,1 ; 0)$ superconformal symmetry. The bosonic symmetries are $S L(2, \mathbf{R})$ conformal symmetry and $S U(2) \times S U(2) R$-symmetry arising from spatial rotations and the $R$-symmetry of $\mathcal{N}=2$ supergravity.
\end{abstract}

November 1999 


\section{Contents}

1. Introduction . . . . . . . . . . . . . . . . . . . . . . . . . . . . . . . . . 1

2. The Multi-Black Hole Moduli Space . . . . . . . . . . . . . . . . . . . . . . . 2

2.1. The Moduli Space Metric . . . . . . . . . . . . . . . . . . . . . . . 3

2.2. The Near-Horizon Limit . . . . . . . . . . . . . . . . . . . . . . . . . . 4

3. $\mathcal{N}=4$ Supersymmetry and the Black Hole Moduli Space . . . . . . . . . . . . . . 4

3.1. Supersymmetry Transformations . . . . . . . . . . . . . . . . . . . . . 5

3.2. Supersymmetric Actions . . . . . . . . . . . . . . . . . . . . . . . . . . 6

4. Superconformal Symmetry with Fermion Multiplets . . . . . . . . . . . . . . . . 8

4.1. Conformal Transformations . . . . . . . . . . . . . . . . . . . . . . . 8

4.2. Conformally Invariant $\mathcal{N}=1$ Actions . . . . . . . . . . . . . . . . . . . . . 11

4.3. $D(2,1 ; \alpha)$ Quantum Mechanics with Fermion Multiplets . . . . . . . . . . . . . 13

5. Superconformal Symmetry of the Near-Horizon Moduli Space . . . . . . . . . . . . 15

Appendix A. A Construction of $d=1$ Superconformal Algebras . . . . . . . . . . . . 18

Appendix B. The $O s p(1 \mid 2)$ Algebra . . . . . . . . . . . . . . . . . . . . . . . . . . . 21

\section{Introduction}

The quantum mechanics of $N$ slowly-moving, four-dimensional extremal ReissnerNordstrom black holes is a sigma model whose target space is the moduli space of multiblack hole solutions. This moduli space is parameterized by the $3 N$ coordinates of the $N$ black holes in $\mathbf{R}^{3}$. The metric on this moduli space was discovered over a decade ago by Ferrell and Eardley [1,2]. When embedded in $\mathcal{N}=2$ supergravity, the static black hole configurations preserve four of the eight supersymmetries. One therefore expects an $\mathcal{N}=4$ supersymmetric quantum mechanics with $4 N$ fermions arising as goldstinos. Typically four supersymmetries would imply a complex structure on the target space. This is clearly not possible in this instance, however, because the dimension of the moduli space can be odd. This puzzle is discussed in [3 35]. Aspects of this problem are explored in [6-8].

In this paper we resolve this puzzle by coupling $3 N$ real $\mathcal{N}=1$ supermultiplets $\Phi^{\mu}$, containing one fermion and one boson apiece, to $N$ real fermionic $\mathcal{N}=1$ supermultiplets $\psi^{A}$, each containing one physical fermion and an auxiliary boson. This gives the required $3 N$ bosons and $4 N$ fermions. We show that taken together these comprise a constrained $\mathcal{N}=4$ multiplet, which is then used to construct a general class of $\mathcal{N}=4$ actions. The geometry of such theories is a generalization of the weak hyperkähler with torsion geometry [4] to $3 N$ dimensions, in which the $S U(2)$ generators of $\mathbf{R}^{3}$ spatial rotations play the role of the triplet of complex structures. The Ferrell-Eardley moduli space is shown to be an 
example of such a geometry and therefore admits $\mathcal{N}=4$ supersymmetry, although we will see that the moduli space metric is modified when the auxiliary bosons are integrated out. Supersymmetry therefore requires corrections to the Ferrell-Eardley metric.

We further consider a near-coincident or near-horizon limit of the moduli space in which the coordinate separation (in spatially conformal coordinates) of the center of mass of the black holes is small compared to their size. In this limit the actual geodesic distance between horizons remains infinite and all curvatures remain small, so the semiclassical approximation is expected to be valid. At low energies this near-horizon quantum mechanics completely decouples from quantum mechanics of widely-separated black holes. We show that the near-horizon theory has an enhanced $D(2,1 ; 0)$ superconformal symmetry. One of the bosonic $S U(2)$ subgroups of $D(2,1 ; 0)$ arises from spacetime rotations, while the other arises form the $R$-symmetry of $\mathcal{N}=2$ supergravity in four dimensions.

One motivation for this work is to understand the spectrum of black hole bound states. The wave function for any state of the near-horizon theory has coordinate separations between black holes which are small compared to their size. Such states are therefore multi-black hole bound states. We expect that the $D(2,1 ; 0)$ superconformal symmetry will play a key role in understanding the bound state spectrum.

In section 2 we describe the moduli space and its near-horizon limit. In section 3 we construct the $\mathcal{N}=4$ supersymmetric extension. In section 4 we describe $D(2,1 ; \alpha)$ superconformal quantum mechanics. In section 5 we show that the near-horizon theory has $D(2,1 ; 0)$ superconformal symmetry. Related work in five dimensions appears in 9 12]. Related work on supersymmetric and superconformal quantum mechanics appears in [3, 1, 13, 16]. Some aspects of sections 3 and 4 have been investigated independently by G. Papadopoulos [17].

\section{The Multi-Black Hole Moduli Space}

We wish to study the moduli space of extremal black hole solutions of pure $\mathcal{N}=2$ supergravity in four dimensions. To this end we will first review results for the moduli space of black hole solutions in Einstein-Maxwell theory, which is the bosonic sector of the supergravity theory under consideration. 


\subsection{The Moduli Space Metric}

The study of black hole moduli spaces was pioneered by Ferrell and Eardley [1], who considered extremally charged black holes in four-dimensional Einstein-Maxwell theory, which has the action

$$
S_{E M}=\frac{1}{16 \pi} \int d^{4} x \sqrt{-g}\left(R-F^{2}\right) .
$$

This theory admits static multi-black hole solutions whose metric and potential are given by

$$
d s^{2}=-\psi^{-2} d t^{2}+\psi^{2} d \mathbf{x}^{2}, \quad A=-\left(1-\psi^{-1}\right) d t
$$

in terms of a harmonic function

$$
\psi(\mathbf{x})=1+\sum_{A} \frac{m_{A}}{\left|\mathbf{x}-\mathbf{x}^{A}\right|}
$$

where $A=1, \ldots, N$ labels the $N$ black holes with masses $m_{A}$ and positions $\mathbf{x}^{A}$. The Einstein-Maxwell action evaluated for solution (2.2)

$$
\mathcal{L}_{0}=-\sum_{A} m_{A}
$$

is independent of $\mathbf{x}^{A}$, thus the positions of the black holes are moduli. This is the well known fact that the electric repulsion and gravitational attraction of extremal black holes cancel. If one gives the black holes small velocities $\mathbf{v}^{A}$ and expands the Einstein-Maxwell action to $\mathcal{O}\left(v^{2}\right)$ around the static solution (2.2) one finds Ferrell and Eardley's effective lagrangian [1]

$$
\mathcal{L}=\frac{1}{2} \sum_{A} m_{A}\left(v^{A}\right)^{2}+\mathcal{L}_{\text {int }}
$$

where

$$
\mathcal{L}_{\text {int }}=\frac{3}{8 \pi} \int d^{3} x \psi^{2} \sum_{A, B} \frac{m_{A} m_{B}}{r_{A}^{3} r_{B}^{3}}\left[\frac{1}{2}\left(\mathbf{r}_{A} \cdot \mathbf{r}_{B}\right)\left|\mathbf{v}^{A}-\mathbf{v}^{B}\right|^{2}-\left(\mathbf{r}_{A} \times \mathbf{r}_{B}\right) \cdot\left(\mathbf{v}^{A} \times \mathbf{v}^{B}\right)\right]
$$

$\mathbf{r}_{A}=\mathbf{x}-\mathbf{x}^{A}$ and $r_{A}=\left|\mathbf{r}_{A}\right|$. It is a curious (and unexplained) fact that (2.6) contains only two-body, three-body and four-body interactions. A very useful form of the effective lagrangian $(2.5)$ is

$$
\mathcal{L}=\frac{1}{2} \sum_{A, B} v^{A k} v^{B l}\left(\delta_{k}^{i} \delta_{l}^{j}+\epsilon^{m i}{ }_{k} \epsilon_{m}{ }^{j}{ }_{l}\right) \partial_{A i} \partial_{B j} L, \quad L=-\frac{1}{16 \pi} \int d^{3} x \psi^{4}
$$

with $\psi$ given by $(2.3)$ and spatial indices $i, j=1,2,3$. 


\subsection{The Near-Horizon Limit}

Let us first consider the single black hole solutions of Einstein-Maxwell theory. For an extremal black hole at $\mathbf{x}^{1}$ the metric takes the form (2.2) where $\psi(\mathbf{x})=1+\frac{m}{\left|\mathbf{x}-\mathbf{x}^{1}\right|}$. The near-horizon limit is defined by $\left|\mathbf{x}-\mathbf{x}^{1}\right| \ll 1$, in which case the metric takes the form

$$
d s^{2}=-\left(\frac{r}{m}\right)^{2} d t^{2}+\left(\frac{m}{r}\right)^{2} d r^{2}+m^{2} d \Omega^{2}
$$

where $r=\left|\mathbf{x}-\mathbf{x}^{1}\right|$. Thus the near-horizon geometry of a single extremal black hole is $A d S_{2} \times S_{2}$.

This analysis of the near-horizon limit of the physical geometry motivates the definition of a similar limit for the multi-black hole moduli space geometry. We require that $\left|\mathbf{x}^{A}-\mathbf{x}^{B}\right| \ll 1$ for $A, B=1, \ldots, N$. In this limit the 1 in the harmonic function $\psi$ can be dropped and the potential in (2.7) is replaced by

$$
L=-\frac{1}{16 \pi} \int d^{3} x\left[\sum_{A} \frac{m_{A}}{\left|\mathbf{x}-\mathbf{x}^{A}\right|}\right]^{4} .
$$

The geometry of the moduli space is still quite complicated. An important feature of this geometry is the existence of noncompact, asymptotically locally flat regions for $\mathbf{x}^{A} \rightarrow \mathbf{x}^{B}$. These correspond to near-coincident black holes.

\section{3. $\mathcal{N}=4$ Supersymmetry and the Black Hole Moduli Space}

In this section we demonstrate that (2.7) admits an $\mathcal{N}=4$ supersymmetric extension. Such an extension is expected because the solution (2.2) preserves four supersymmetries when embedded in $\mathcal{N}=2, D=4$ supergravity. The four broken supersymmetries are expected to lead to four goldstinos per black hole, and hence $4 N$ fermions in addition to the $3 N$ bosons in the supersymmetric quantum mechanics. Since the unbroken supercharges transform under spatial rotations, we further expect an $S U(2) R$ symmetry with the bosons in triplets and the fermions in doublets. A second $S U(2)$ quantum mechanical $R$-symmetry, with singlet bosons and doublet fermions, is expected from reduction of the four-dimensional $R$-symmetry.

This field content sounds rather exotic as one usually encounters equal numbers of bosons and fermions in a supersymmetric theory. However in one dimension there is a fermion supermultiplet $\Psi$ whose only physical fields are fermions [18]. Accordingly in this 
section we consider $3 N$ real $\mathcal{N}=1$ multiplets $\Phi^{\mu}$ - each of which contains one boson and one fermion - along with $N$ extra fermion multiplets $\Psi^{A}$ - each of which contains a single physical fermion. These are combined into a constrained $\mathcal{N}=4$ multiplet in a manner which properly realizes the $R$-symmetries. Invariant actions are then constructed using $\mathcal{N}=4$ superspace. This construction is found to include the black hole quantum mechanics as a special case.

\subsection{Supersymmetry Transformations}

Our treatment of supersymmetry follows closely that of Coles and Papadopoulos [18], although our notation differs very slightly. We first introduce the $\mathcal{N}=1$ superfields

$$
\Phi^{\mu}=X^{\mu}-i \theta \lambda^{\mu}, \quad \Psi^{A}=i \psi^{A}+i \theta b^{A},
$$

where $X^{\mu}$ and $b^{A}$ are real bosons and $\lambda^{\mu}$ and $\psi^{A}$ are real fermions. The bosonic superfield $\Phi^{\mu}$ is the usual map from $\mathcal{N}=1$ superspace into the sigma model manifold $\mathcal{M}$. We define the usual superspace derivatives for the $0^{\text {th }}$ supersymmetry

$$
\mathcal{Q}=\frac{d}{d \theta}+i \theta \frac{d}{d t}, \quad \mathcal{D}=\frac{d}{d \theta}-i \theta \frac{d}{d t}
$$

which obey

$$
\mathcal{Q}^{2}=i \frac{d}{d t}, \quad \mathcal{D}^{2}=-i \frac{d}{d t}, \quad\{\mathcal{Q}, \mathcal{D}\}=0
$$

For the remaining supersymmetries, $i=1, \ldots, \mathcal{N}-1$, one makes the ansatz

$$
\mathcal{Q}_{i} \Phi^{\mu}=I_{i}{ }^{\mu}{ }_{\nu} \mathcal{D} \Phi^{\nu}+e_{i}{ }^{\mu}{ }_{A} \Psi^{A}, \quad \mathcal{Q}_{i} \Psi^{A}=I_{i}{ }^{A}{ }_{B} \mathcal{D} \Psi^{B}-i e_{i}{ }^{A}{ }_{\mu} \dot{\Phi}^{\mu}
$$

where ${ }^{\cdot}=\frac{d}{d t}$. Under an infinitesimal supersymmetry transformation parametrized by $\zeta^{r}$, $r=0, \ldots, \mathcal{N}-1$, the superfields $\Phi^{\mu}$ and $\Psi^{A}$ transform as

$$
\delta_{\zeta} \Phi^{\mu}=\zeta^{r} \mathcal{Q}_{r} \Phi^{\mu}, \quad \delta_{\zeta} \Psi^{A}=\zeta^{r} \mathcal{Q}_{r} \Psi^{A}
$$

where $\mathcal{Q}_{0} \equiv \mathcal{Q}$. The conditions that $(3.2)$ and $(3.4)$ close to the supersymmetry algebra

$$
\left\{\mathcal{Q}_{r}, \mathcal{Q}_{s}\right\}=2 i \delta_{r s} \frac{d}{d t}, \quad r, s=0, \ldots, \mathcal{N}-1
$$

appear in Appendix A of [18] (and in much more generality than our special case). The most interesting constraints are

$$
-I_{i}{ }^{\mu}{ }_{\nu} I_{i}{ }_{\rho}{ }_{\rho}+e_{i}{ }^{\mu}{ }_{A} e_{i}{ }^{A}{ }_{\rho}=\delta_{\rho}^{\mu}, \quad \mathcal{N}\left(I_{i}\right)^{\mu}{ }_{\nu \rho}=0
$$


for all $i$, where $\mathcal{N}\left(I_{i}\right)$ is the Nijenhuis tensor of $I_{i}$.

If the $e_{i}$ terms are absent (3.7) requires the $I_{i}$ to be complex structures. This is impossible, however, if the target space is $3 N$ dimensional as in the case of current interest. A simple solution of the constraints when $\mu=1, \ldots, 3 N$ and $A=1, \ldots, N$ is

$$
\left(I_{i}\right)^{A j}{ }_{B k}=\delta_{B}^{A} \epsilon_{i}{ }^{j}{ }_{k}, \quad\left(I_{i}\right)^{A}{ }_{B}=0, \quad\left(e_{i}\right)^{A}{ }_{B j}=\delta_{B}^{A} \delta_{i j}, \quad\left(e_{i}\right)^{B j}{ }_{A}=\delta_{A}^{B} \delta_{i}^{j},
$$

where we have replaced the index $\mu$ with the index pair $A i$ with $i=1,2,3$. It is easy to check directly that the closure conditions (3.6) are satisfied by (3.8).

\subsection{Supersymmetric Actions}

In order to construct supersymmetric actions it is efficient to introduce constrained $\mathcal{N}=4$ superfields. We employ anticommuting superspace coordinates $\theta_{r}, r=0, \ldots, 3$, where $\theta_{0} \equiv \theta$ is the usual $\mathcal{N}=1$ superspace coordinate. The corresponding $\mathcal{N}=4$ superfields are denoted in boldface $\boldsymbol{\Phi}^{\mu}\left(t, \theta_{r}\right)$ and $\boldsymbol{\Psi}^{A}\left(t, \theta_{r}\right)$. Their $\theta_{i}$-independent components are the usual $\mathcal{N}=1$ superfields $\Phi^{\mu}(t, \theta)$ and $\Psi^{A}(t, \theta)$ appearing in (3.1). We define

$$
\mathcal{Q}_{r}=\frac{d}{d \theta_{r}}+i \theta_{r} \frac{d}{d t}, \quad \mathcal{D}_{r}=\frac{d}{d \theta_{r}}-i \theta_{r} \frac{d}{d t}
$$

which obey

$$
\left\{\mathcal{Q}_{r}, \mathcal{Q}_{s}\right\}=2 i \delta_{r s} \frac{d}{d t}=-\left\{\mathcal{D}_{r}, \mathcal{D}_{s}\right\}, \quad\left\{\mathcal{Q}_{r}, \mathcal{D}_{s}\right\}=0
$$

with $\mathcal{D}_{0} \equiv \mathcal{D}$. The supersymmetry transformations in $\mathcal{N}=4$ superspace are generated by the $\mathcal{Q}_{r}$,

$$
\delta_{\zeta} \boldsymbol{\Phi}^{\mu}=\zeta^{r} \mathcal{Q}_{r} \boldsymbol{\Phi}^{\mu}, \quad \delta_{\zeta} \Psi^{A}=\zeta^{r} \mathcal{Q}_{r} \Psi^{A}
$$

which automatically obey the supersymmetry algebra. The $\mathcal{N}=4$ superfields have many fermionic components which we need to reduce in number by a constraint. At the same time we wish to recover the transformations (3.4). Both of these goals are accomplished by the constraint

$$
\begin{aligned}
\mathcal{D}_{i} \boldsymbol{\Phi}^{A j} & =\epsilon_{i}{ }_{k}{ }_{k} \mathcal{D} \boldsymbol{\Phi}^{A k}+\delta_{i}^{j} \boldsymbol{\Psi}^{A} \\
\mathcal{D}_{i} \boldsymbol{\Psi}^{A} & =-i \delta_{i j} \dot{\boldsymbol{\Phi}}^{A j}
\end{aligned}
$$

One recovers (3.4) by plugging these constraints into the $\theta_{i}$-independent part of (3.11).

A manifestly $\mathcal{N}=4$ supersymmetric action can now be constructed as

$$
S=\frac{1}{2} \int d t d^{4} \theta L(\mathbf{\Phi}) .
$$


This can be reduced to an $\mathcal{N}=1$ superspace action using the constraints 3.12 and integrating over the three $\theta_{i}$. Using $\int d \theta_{i} \mathbf{F}=\left.\mathcal{D}_{i} \mathbf{F}\right|_{\theta_{i}=0}+($ total derivative $)$ we find that

$$
\begin{aligned}
S=\frac{1}{2} \int d t d \theta\left[L_{, \mu \nu \rho}\right. & \mathcal{D}_{1} \boldsymbol{\Phi}^{\mu} \mathcal{D}_{2} \boldsymbol{\Phi}^{\nu} \mathcal{D}_{3} \boldsymbol{\Phi}^{\rho} \\
& +L_{, \mu \nu}\left(\mathcal{D}_{1} \boldsymbol{\Phi}^{\mu} \mathcal{D}_{2} \mathcal{D}_{3} \boldsymbol{\Phi}^{\nu}+\mathcal{D}_{2} \boldsymbol{\Phi}^{\mu} \mathcal{D}_{3} \mathcal{D}_{1} \boldsymbol{\Phi}^{\nu}+\mathcal{D}_{3} \boldsymbol{\Phi}^{\mu} \mathcal{D}_{1} \mathcal{D}_{2} \boldsymbol{\Phi}^{\nu}\right) \\
& \left.+L_{, \mu} \mathcal{D}_{1} \mathcal{D}_{2} \mathcal{D}_{3} \boldsymbol{\Phi}^{\mu}\right]_{\theta_{i}=0}
\end{aligned}
$$

where $\mu, \nu, \rho=1, \ldots, 3 N$ run over the moduli space indices $A j$. From (3.12) we see that for $k \neq i$

$$
\begin{gathered}
\mathcal{D}_{k} \mathcal{D}_{i} \boldsymbol{\Phi}^{A j}=-\mathcal{D}_{i} \mathcal{D}_{k} \boldsymbol{\Phi}^{A j}=-\epsilon_{i}{ }_{k} \mathcal{D} \boldsymbol{\Psi}^{A}-2 i \delta_{m[k} \delta_{i]}{ }^{j} \dot{\boldsymbol{\Phi}}^{A m} \\
\mathcal{D}_{1} \mathcal{D}_{2} \mathcal{D}_{3} \boldsymbol{\Phi}^{\mu}=-i \mathcal{D} \dot{\boldsymbol{\Phi}}^{\mu}
\end{gathered}
$$

We may write the action in a more symmetric form by anticommuting and integrating the third line of (3.14) by parts:

$$
\begin{aligned}
S=\frac{1}{2} \int d t d \theta\left[\frac{1}{3 !} L_{, \mu \nu \rho} \epsilon^{i j k} \mathcal{D}_{i} \boldsymbol{\Phi}^{\mu} \mathcal{D}_{j} \boldsymbol{\Phi}^{\nu} \mathcal{D}_{k} \boldsymbol{\Phi}^{\rho}\right. & \\
& \left.+L_{, \mu \nu}\left(\frac{1}{2} \epsilon^{i j k} \mathcal{D}_{i} \boldsymbol{\Phi}^{\mu} \mathcal{D}_{j} \mathcal{D}_{k} \boldsymbol{\Phi}^{\nu}+i \mathcal{D} \boldsymbol{\Phi}^{\mu} \dot{\boldsymbol{\Phi}}^{\nu}\right)\right]_{\theta_{i}=0}
\end{aligned}
$$

The most general supersymmetric action for $\Phi^{\mu}$ and $\Psi^{A}$ contains the terms 18]

$$
\begin{aligned}
S=\int d t d \theta\left[\frac{i}{2} g_{\mu \nu} \mathcal{D} \Phi^{\mu} \dot{\Phi}^{\nu}-\frac{1}{2} h_{A B} \Psi^{A} \mathcal{D} \Psi^{B}-i f_{\mu A} \dot{\Phi}^{\mu} \Psi^{A}+\frac{1}{3 !} c_{\mu \nu \rho} \mathcal{D} \Phi^{\mu} \mathcal{D} \Phi^{\nu} \mathcal{D} \Phi^{\rho}\right. \\
\left.+\frac{1}{2 !} n_{\mu \nu A} \mathcal{D} \Phi^{\mu} \mathcal{D} \Phi^{\nu} \Psi^{A}+\frac{1}{2 !} m_{\mu A B} \mathcal{D} \Phi^{\mu} \Psi^{A} \Psi^{B}+\frac{1}{3 !} l_{A B C} \Psi^{A} \Psi^{B} \Psi^{C}\right]
\end{aligned}
$$

Using (3.12) and (3.15) we can read off the quadratic terms of (3.17),

$$
\begin{gathered}
g_{A i B j}=\left(\delta_{i}^{k} \delta_{j}^{l}+\epsilon^{m k}{ }_{i} \epsilon_{m}{ }^{l}{ }_{j}\right) \partial_{A k} \partial_{B l} L \\
h_{A B}=\delta^{i j} \partial_{A i} \partial_{B j} L \\
f_{A i B}=\epsilon^{j k}{ }_{i} \partial_{A j} \partial_{B k} L .
\end{gathered}
$$

The $\mathcal{D} \Psi \mathcal{D} \Phi$ term has been integrated by parts and absorbed into the $f$ and $n$ terms. The cubic terms are

$$
c_{A i B j C k}=\frac{1}{2} \epsilon^{p q h} \epsilon_{p}{ }_{i} \epsilon_{q}{ }^{m}{ }_{j} \epsilon_{h}{ }^{n}{ }_{k} \partial_{A l} \partial_{B m} \partial_{C n} L
$$




$$
\begin{gathered}
n_{A i B j C}=\frac{1}{2}\left(\epsilon^{p q n} \epsilon_{p}{ }^{l}{ }_{i} \epsilon_{q}{ }^{m}{ }_{j}+\epsilon_{i}{ }_{i}{ }^{n} \delta_{j}^{m}-\epsilon^{m}{ }_{j}{ }^{n} \delta_{i}^{l}\right) \partial_{A l} \partial_{B m} \partial_{C n} L, \\
m_{A i B C}=\frac{1}{2} \epsilon^{j m n} \epsilon_{j}{ }^{l}{ }_{i} \partial_{A l} \partial_{B m} \partial_{C n} L \\
l_{A B C}=\frac{1}{2} \epsilon^{l m n} \partial_{A l} \partial_{B m} \partial_{C n} L .
\end{gathered}
$$

The actions (3.13) and (3.18) are $\mathcal{N}=4$ supersymmetric for any function $L$. Comparing the bosonic metric (3.19) appearing in the action with the moduli space metric (2.7) we conclude that the choice

$$
L=-\frac{1}{16 \pi} \int d^{3} x \psi^{4}
$$

describes the $\mathcal{N}=4$ supersymmetric quantum mechanics of $N$ black holes.

It is straightforward to check that with $L$ given by (3.26), the coupling $f_{\mu A}$ computed from (3.21) is nonzero (as long as $N>2$ ). From (3.18) it can be seen that this implies a coupling of the form $f_{\mu A} \dot{X}^{\mu} b^{A}$ once the superfields are written out in terms of components. When the auxiliary fields $b^{A}$ are integrated out, the quadratic action $g_{\mu \nu} \dot{X}^{\mu} \dot{X}^{\nu}$ will receive an additional contribution proportional to $f_{\mu A} f_{\nu B} h^{A B} \dot{X}^{\mu} \dot{X}^{\nu}$. This signifies a modification of the moduli space metric required by supersymmetry.

\section{Superconformal Symmetry with Fermion Multiplets}

In this section we investigate the superconformal extension of the supersymmetry algebra developed in the previous section. We continue to work with the multiplets $\left(X^{\mu}, \lambda^{\mu}\right)$ and $\left(\psi^{A}, b^{A}\right)$. In sections 4.1 and 4.2 we will work with generic $I_{i}$ and $e_{i}$, requiring only that the extra supersymmetries (3.4) satisfy the supersymmetry algebra (3.6) - we will restrict our attention to the specific choices of $I_{i}$ and $e_{i}$ (3.8) only in section 4.3. Although our approach will resemble that of [9] we will work entirely in the lagrangian formulation. One consequence of this is that we will investigate separately the closure of the superconformal algebra on the fields $\left(X^{\mu}, \lambda^{\mu}, \psi^{A}, b^{A}\right)$ and invariance of the action (3.14).

\subsection{Conformal Transformations}

We investigate the behavior of the fields $\left(X^{\mu}, \lambda^{\mu}, \psi^{A}, b^{A}\right)$ under conformal transformations. It is convenient to parametrize a conformal transformation by

$$
\epsilon(t)=\epsilon_{H}+2 t \epsilon_{D}+t^{2} \epsilon_{K}
$$


where $\epsilon_{H}, \epsilon_{D}$, and $\epsilon_{K}$ are constant infinitesimal parameters corresponding respectively to time translations, dilatations and special conformal transformations. With this parametrization the $S L(2, \mathbf{R})$ algebra takes the form

$$
\left[\delta_{\epsilon_{1}}, \delta_{\epsilon_{2}}\right]=\delta_{\left[\epsilon_{1}, \epsilon_{2}\right]}, \quad \text { where }\left[\epsilon_{1}, \epsilon_{2}\right]=\epsilon_{1} \dot{\epsilon}_{2}-\epsilon_{2} \dot{\epsilon}_{1}
$$

If we define the generators $H, D$ and $K$ by

$$
\delta_{\epsilon_{H}}=i \epsilon_{H} H, \quad \delta_{2 \epsilon_{D} t}=i \epsilon_{D} D, \quad \delta_{\epsilon_{K} t^{2}}=i \epsilon_{K} K,
$$

then the algebra takes the familiar form

$$
[H, K]=-i D, \quad[H, D]=-2 i H, \quad[K, D]=2 i K
$$

The variation of the field $X^{\mu}$ under a conformal transformation is

$$
\delta_{\epsilon} X^{\mu}=-\epsilon \dot{X}^{\mu}+\frac{1}{2} \dot{\epsilon} D^{\mu}
$$

for some vector field $D^{\mu}(X)$. One easily checks that the $S L(2, \mathbf{R})$ algebra (4.2) is satisfied for any $D^{\mu}$. For the remaining fields, we make the ansätze 1

$$
\begin{aligned}
\delta_{\epsilon} \lambda^{\mu} & =-\epsilon \dot{\lambda}^{\mu}+\frac{1}{2} \dot{\epsilon} F^{\mu}, \\
\delta_{\epsilon} \psi^{A} & =-\epsilon \dot{\psi}^{A}+\frac{1}{2} \dot{\epsilon} G^{A}, \\
\delta_{\epsilon} b^{A} & =-\epsilon \dot{b}^{A}+\frac{1}{2} \dot{\epsilon} H^{A} .
\end{aligned}
$$

Again one easily checks that the $S L(2, \mathbf{R})$ algebra is satisfied as long as $F^{\mu}(X, \lambda), G^{A}(X, \psi)$ and $H^{A}(X, b)$ do not depend on the time derivatives of the four basic fields.

We now wish to enlarge the algebra to include the supersymmetries (3.2) and (3.4). In analogy with the above discussion we will express the supersymmetry variations in terms of generators $Q^{r}$ of supersymmetry transformations on the component fields (not to be confused with the superderivatives (3.9), which act on superfields) defined by

$$
\delta_{\zeta}=i \zeta_{r} Q^{r}
$$

1 Although we shall not find it necessary, one could consider more general conformal transformations where $D^{\mu}, F^{\mu}, G^{A}$ and $H^{A}$ are functions of $\left(X^{\mu}, \lambda^{\mu}, \psi^{A}, b^{A}\right)$. In this case the conditions required by closure of the $O s p(1 \mid 2)_{r}$ algebras become somewhat more complicated. 
where the $\zeta_{r}$ are anticommuting parameters. Since the supersymmetry transformations do not involve the index $A$ we will suppress this index and consider just the fields $\left(X^{i}, \lambda^{i}, \psi, b\right)$. The action of these generators on the component fields is

$$
Q^{0} X^{i}=-\lambda^{i}, \quad Q^{0} \lambda^{i}=-i \dot{X}^{i}, \quad Q^{0} \psi=-i b, \quad Q^{0} b=-\dot{\psi}
$$

and

$$
\begin{aligned}
Q^{i} X^{j} & =-\epsilon^{i j}{ }_{k} \lambda^{k}+\delta^{i j} \psi, \\
Q^{i} \lambda^{j} & =i \epsilon^{i j}{ }_{k} \dot{X}^{k}-i \delta^{i j} b, \\
Q^{i} \psi & =i \dot{X}^{i}, \\
Q^{i} b & =-\dot{\lambda}^{i} .
\end{aligned}
$$

It is straightforward to verify that these generators obey the required anticommutation relations $\left\{Q^{r}, Q^{s}\right\}=2 \delta^{r s} H$.

We first enlarge the $S L(2, \mathbf{R})$ algebra to $O s p(1 \mid 2)_{0}$ by incorporating the $\mathcal{N}=1$ supersymmetry transformation $Q^{0}$. Following the procedure outlined in Appendix A we define the superconformal generator $S^{0}$ by the relation $\left[K, Q^{0}\right]=i S^{0}$. The requirement that $\left[D, Q^{0}\right]=i Q^{0}$ on $X^{\mu}$ implies the unique choice $F^{\mu}=Q^{0}\left(X^{\mu}-D^{\mu}\right)$. This same relation on $\psi^{A}$ requires $H^{A}=i Q^{0}\left(G^{A}-\psi^{A}\right)$. The relation $\left[K, S^{0}\right]=0$ gives no additional constraints, so the remaining (anti-) commutation relations of $O s p(1 \mid 2)_{0}$ (see Appendix B) follow with no additional restrictions on $D^{\mu}(X)$ or $G^{A}(X, \psi)$.

The next step is to incorporate the extended supersymmetries (4.9). We first examine the conditions required by closure of the $O s p(1 \mid 2)_{i}$ subalgebra, which is generated by $\left(H, D, K, Q^{i}, S^{i}\right)$, for each value of $i$. We define $S^{i}$ by $\left[K, Q^{i}\right]=i S^{i}$ as before. We find

$$
\left[D, Q^{i}\right] X^{\mu}=i Q^{i} X^{\mu}+i\left(\mathcal{L}_{D} I^{i \mu}{ }_{\nu}\right) \lambda^{\nu}-\psi^{A}\left(\mathcal{L}_{D} e^{i \mu}{ }_{A}+e^{i \mu}{ }_{A}\right)-e^{i \mu}{ }_{A} G^{A} .
$$

so that closure of the algebra requires $\mathcal{L}_{D} I_{i}{ }^{\mu}{ }_{\nu}=0$. Furthermore, if we make the ansatz that $\left(\mathcal{L}_{D}-\beta\right) e_{i}{ }^{\mu}{ }_{A}=0$ for some constant $\beta$ then (4.10) requires

$$
G^{A}=-(\beta+1) \psi^{A} .
$$

Acting on $\psi^{A}$ we find

$$
\left[D, Q^{i}\right] \psi^{A}=i Q^{i} \psi^{A}+\dot{X}^{\mu}\left(\mathcal{L}_{D}+\beta\right) e_{i}{ }^{A}{ }_{\mu}
$$

so we must have

$$
\left(\mathcal{L}_{D}+\beta\right) e_{i}{ }^{A}{ }_{\mu}=0 .
$$


The rest of the $\operatorname{Osp}(1 \mid 2)_{i}$ algebra follows without further restrictions.

We have verified that the supersymmetry transformations (3.2) and (3.4) together with the conformal transformations

$$
\begin{aligned}
\delta_{\epsilon} X^{\mu} & =-\epsilon \dot{X}^{\mu}+\frac{1}{2} \dot{\epsilon} D^{\mu}, \\
\delta_{\epsilon} \lambda^{\mu} & =-\epsilon \dot{\lambda}^{\mu}+\frac{1}{2} \dot{\epsilon}\left(D^{\mu}{ }_{, \nu} \lambda^{\nu}-\lambda^{\mu}\right), \\
\delta_{\epsilon} \psi^{A} & =-\epsilon \dot{\psi}^{A}-\frac{1}{2} \dot{\epsilon}(\beta+1) \psi^{A}, \\
\delta_{\epsilon} b^{A} & =-\epsilon \dot{b}^{A}-\frac{1}{2} \dot{\epsilon}(\beta+2) b^{A}
\end{aligned}
$$

satisfy the $\mathcal{N}$ separate algebras $O s p(1 \mid 2)_{r}$ as long as

$$
\mathcal{L}_{D} I_{i}{ }^{\mu}{ }_{\nu}=\left(\mathcal{L}_{D}-\beta\right) e_{i}{ }^{\mu}{ }_{A}=\left(\mathcal{L}_{D}+\beta\right) e_{i}{ }^{A}{ }_{\mu}=0
$$

for each $i=1, \ldots, \mathcal{N}-1$.

We must now knit these $\mathcal{N} O s p(1 \mid 2)_{r}$ algebras together into the appropriate superalgebra. Following the procedure outlined in Appendix A, it remains only to check that the $Q^{r}$ lie in an appropriate spinor representation of the $R$ symmetry algebra which appears on the right-hand side of the $\left\{Q^{r}, S^{s}\right\}$ anticommutator. This will be done for the constant $I$ and $e$ case (3.8) in section 4.3 below.

\subsection{Conformally Invariant $\mathcal{N}=1$ Actions}

In this section we construct conformally invariant actions out of the $\mathcal{N}=1$ multiplets $\Phi^{\mu}$ and $\Psi^{A}$. Let us start with the superfield $\Phi^{\mu}$. The most general action involving only dimensionless couplings is [18]

$$
S_{1}=\int d t d \theta \frac{i}{2} g_{\mu \nu} \mathcal{D} \Phi^{\mu} \dot{\Phi}^{\nu}+\frac{1}{6} c_{\mu \nu \rho} \mathcal{D} \Phi^{\mu} \mathcal{D} \Phi^{\nu} \mathcal{D} \Phi^{\rho}
$$

In terms of component fields

$$
S_{1}=\int d t \frac{1}{2} g_{\mu \nu}\left[\dot{X}^{\mu} \dot{X}^{\nu}+i \lambda^{\mu} D_{t} \lambda^{\nu}\right]+\frac{i}{2} c_{\mu \nu \rho} \lambda^{\mu} \lambda^{\nu} \dot{X}^{\rho}-\frac{1}{6} c_{\mu \nu \rho, \sigma} \lambda^{\mu} \lambda^{\nu} \lambda^{\rho} \lambda^{\sigma},
$$

where

$$
D_{t} \lambda^{\nu}=\dot{\lambda}^{\nu}+\Gamma_{\rho \sigma}^{\nu} \dot{X}^{\rho} \lambda^{\sigma}
$$

It is convenient to consider separately the bosonic and fermionic terms in (4.17). Using (4.14) we find

$$
\delta_{\epsilon}\left(\frac{1}{2} g_{\mu \nu} \dot{X}^{\mu} \dot{X}^{\nu}\right) \sim \frac{1}{4} \dot{\epsilon}\left[\left(\mathcal{L}_{D}-2\right) g_{\mu \nu}\right] \dot{X}^{\mu} \dot{X}^{\nu}+\frac{1}{2} \ddot{\epsilon} D_{\mu} \dot{X}^{\mu}
$$


where $\sim$ denotes equality up to total derivatives. Thus invariance of this term under dilatations requires

$$
\left(\mathcal{L}_{D}-2\right) g_{\mu \nu}=0
$$

i.e. that $D^{\mu}$ is a homothety. Invariance under special conformal transformations requires in addition that

$$
D_{\mu}=\partial_{\mu} K
$$

for some function $K$. A vector field $D^{\mu}$ obeying (4.20) and (4.21) is known as a closed homothety. Note that in general invariance of the action under dilatations does not guarantee invariance under the full conformal group. In all further calculations we assume (4.20) and (4.21) hold. For the two-fermion terms in (4.17) we find

$$
\delta_{\epsilon}\left(\frac{i}{2} g_{\mu \nu} \lambda^{\mu} D_{t} \lambda^{\nu}+\frac{i}{2} c_{\mu \nu \rho} \lambda^{\mu} \lambda^{\nu} \dot{X}^{\rho}\right) \sim \frac{i}{4} \dot{\epsilon}\left[\left(\mathcal{L}_{D}-2\right) c_{\mu \nu \rho}\right] \lambda^{\mu} \lambda^{\nu} \dot{X}^{\rho}+\frac{i}{4} \ddot{\epsilon} D^{\mu} c_{\mu \nu \rho} \lambda^{\nu} \lambda^{\rho}
$$

so invariance requires that

$$
\left(\mathcal{L}_{D}-2\right) c_{\mu \nu \rho}=0, \quad D^{\mu} c_{\mu \nu \rho}=0
$$

Again, the first condition is required by dilatation invariance and the second is an additional constraint required for full conformal symmetry. Finally, varying the four-fermion terms in $(4.17)$ gives

$$
\delta_{\epsilon}\left(-\frac{1}{6} c_{\mu \nu \rho, \sigma} \lambda^{\mu} \lambda^{\nu} \lambda^{\rho} \lambda^{\sigma}\right) \sim \frac{1}{12} \dot{\epsilon} \partial_{\sigma}\left[\left(\mathcal{L}_{D}-2\right) c_{\mu \nu \rho}\right] \lambda^{\sigma} \lambda^{\mu} \lambda^{\nu} \lambda^{\rho}
$$

which vanishes as consequence of (4.23), giving no further constraints. The conditions (4.20), (4.21) and (4.23) agree precisely with those found by the authors of [9], who used the Hamiltonian formalism.

When the fermion multiplet $\Psi^{A}=i \psi^{A}+i \theta b^{A}$ is included there are five additional terms that one can add to the superspace lagrangian (4.16),

$$
\begin{aligned}
\mathcal{L}_{2}=-\frac{1}{2} h_{A B} \Psi^{A} \mathcal{D} \Psi^{B} & +\frac{1}{6} l_{A B C} \Psi^{A} \Psi^{B} \Psi^{C} \\
& -i f_{\mu A} \dot{\Phi}^{\mu} \Psi^{A}+\frac{1}{2} m_{\mu A B} \mathcal{D} \Phi^{\mu} \Psi^{A} \Psi^{B}+\frac{1}{2} n_{\mu \nu A} \mathcal{D} \Phi^{\mu} \mathcal{D} \Phi^{\nu} \Psi^{A}
\end{aligned}
$$


The calculation of $\delta_{\epsilon} \mathcal{L}_{2}$ is similar to the above calculation, so we will simply quote the result. The terms (4.25) are dilatation invariant provided that

$$
\begin{aligned}
\mathcal{L}_{D} h_{A B} & =(2 \beta+2) h_{A B}, \\
\mathcal{L}_{D} l_{A B C} & =(3 \beta+2) l_{A B C}, \\
\mathcal{L}_{D} f_{\mu A} & =(\beta+2) f_{\mu A}, \\
\mathcal{L}_{D} m_{\mu A B} & =(2 \beta+2) m_{\mu A B}, \\
\mathcal{L}_{D} n_{\mu \nu A} & =(\beta+2) n_{\mu \nu A},
\end{aligned}
$$

and invariant under special conformal transformations if, in addition,

$$
\begin{aligned}
& 0=D^{\mu} m_{\mu A B}, \\
& 0=D^{\nu}\left(n_{\mu \nu A}-\nabla_{\mu} f_{\nu A}\right) .
\end{aligned}
$$

Note that the $n_{\mu \nu A}$ and $f_{\mu A}$ terms in (4.25) mix under conformal transformations.

\section{3. $D(2,1 ; \alpha)$ Quantum Mechanics with Fermion Multiplets}

In this section we work out the $R$-symmetries and full superconformal algebra for the special case (3.8) with constant $I$ and $e$. We assume the existence of a closed homothety of the form

$$
D^{A i}=\frac{2}{h} X^{A i}
$$

for some constant $h$. Since $e_{i}{ }^{A}{ }_{\mu}$ is now $e_{i}{ }^{A}{ }_{B j}=\delta_{B}^{A} \delta_{i j}$, which is constant, we have

$$
\mathcal{L}_{D} e_{i}{ }^{A}{ }_{\mu}=e_{i}{ }^{A}{ }_{\nu} D^{\nu}{ }_{, \mu}=\frac{2}{h} e_{i}{ }^{A}{ }_{\mu}
$$

Comparing with (4.13) we see that $\beta$ and $h$ must be related by $\beta=-\frac{2}{h}$.

The first step is to find the superconformal generators $S^{r}$, which are defined by $S^{r}=$ $i\left[Q^{r}, K\right]$. Using (4.8) and (4.9), and again suppressing the $A$ index, we find

$$
\begin{array}{rlrl}
S^{0} X^{j} & =-t \lambda^{j}, \\
S^{0} \lambda^{j} & =-i t \dot{X}^{j}+\frac{2 i}{h} X^{j}, & S^{i} X^{j} & =-t \epsilon^{i j}{ }_{k} \lambda^{k}+t \delta^{i j} \psi, \\
S^{0} \psi & =-i t b, & S^{i} \lambda^{j} & =i t \epsilon^{i j}{ }_{k} \dot{X}^{k}-i t \delta^{i j} b-\frac{2 i}{h} \epsilon^{i j}{ }_{k} X^{k}, \\
S^{0} b & =-t \dot{\psi}+\left(\frac{2}{h}-1\right) \psi & =i t \dot{X}^{i}-\frac{2 i}{h} X^{i}, \\
S^{i}, & S^{i} b & =-t \dot{\lambda}^{i}+\left(\frac{2}{h}-1\right) \lambda^{i} .
\end{array}
$$


As per the discussion in Appendix A, the hard part is now to package the $\left\{Q^{r}, S^{s}\right\}$ anticommutator into a nice form by defining the appropriate $R$ symmetry generators. We find that the $\{Q, S\}$ anticommutator has the form

$$
\left\{Q^{r}, S^{s}\right\}=D \delta^{r s}+\frac{4(h+1)}{h}\left(T_{+i}\right)^{r s} R_{+}^{i}-\frac{4}{h}\left(T_{-i}\right)^{r s} R_{-}^{i},
$$

where the $T_{ \pm}^{i}$ are constant matrices defined by

$$
\left(T_{ \pm}^{i}\right)^{r s}=\mp \delta^{i[r} \delta^{s] 0}+\frac{1}{2} \epsilon^{i r s}
$$

and the $R$ symmetry generators are given by

$$
\begin{aligned}
R_{ \pm}^{i} X^{j} & =\frac{i}{2}(1 \mp 1) \epsilon_{k}^{i j} X^{k}, \\
R_{ \pm}^{i} \lambda^{j} & =\frac{i}{2}\left(\epsilon^{i j}{ }_{k} \lambda^{k} \mp \delta^{i j} \psi\right), \\
R_{ \pm}^{i} \psi & = \pm \frac{i}{2} \lambda^{i}, \\
R_{ \pm}^{i} b & =0 .
\end{aligned}
$$

The $T_{ \pm}^{i}$ satisfy

$$
\left[T_{+}^{i}, T_{-}^{j}\right]=0, \quad\left[T_{ \pm}^{i}, T_{ \pm}^{j}\right]=-\epsilon_{k}^{i j} T_{ \pm}^{k}, \quad\left\{T_{ \pm}^{i}, T_{ \pm}^{j}\right\}=-\frac{1}{2} \delta^{i j}
$$

and the $R$ symmetries satisfy

$$
\left[R_{+}^{i}, R_{-}^{j}\right]=0, \quad\left[R_{ \pm}^{i}, R_{ \pm}^{j}\right]=i \epsilon^{i j} R_{ \pm}^{k}
$$

Thus the $R$-symmetry of this theory is $S U(2) \times S U(2)$. The $R_{-}$act on the $X^{A j}$ as an $S O(3)$ triplet, so we interpret this $S U(2)$ symmetry as arising from the $S O(3)$ spatial rotations of the original theory. 2 The $X^{A i}$ are uncharged under $R_{+}$, so the second $S U(2)$ must come from the $S U(2) R$-symmetry of the original $D=4, \mathcal{N}=2$ supergravity. The four $Q$ 's transform as complex doublets of each $S U(2)$,

$$
\left[R_{ \pm}^{i}, Q^{0}\right]= \pm \frac{i}{2} Q^{i}, \quad\left[R_{ \pm}^{i}, Q^{j}\right]=\frac{i}{2}\left(\mp \delta^{i j} Q^{0}+\epsilon^{i j}{ }_{k} Q^{k}\right)
$$

and the $S$ 's similarly. We recognize (4.31) and (4.36) as the defining relations of the $D(2,1 ; \alpha)$ superalgebra with parameter

$$
\alpha=-h-1
$$

In conclusion, we have demonstrated that an $\mathcal{N}=4$ supersymmetric theory with action (3.13) has $D(2,1 ; \alpha=-h-1)$ symmetry if it admits a closed homothety of the form 4.28$)$.

2 A more careful analysis shows that $S O(3)$ spatial rotations are generated by the diagonal subgroup $R_{-}+R_{+}$. 


\section{Superconformal Symmetry of the Near-Horizon Moduli Space}

In this section we demonstrate that the quantum mechanics defined by (2.7) admits a $D(2,1 ; 0)$ symmetry in the near-horizon limit. In the near-horizon limit the metric is

$$
g_{A k B l}=G_{k l}^{i j} \partial_{A i} \partial_{B j} L
$$

where

$$
G_{k l}^{i j}=\delta_{k}^{i} \delta_{l}^{j}+\epsilon_{k}^{m i} \epsilon_{m}^{j}{ }_{l}
$$

and

$$
L\left(x^{A i}\right)=-\frac{1}{16 \pi} \int d^{3} x\left[\sum_{A} \frac{m_{A}}{\left|\mathbf{x}-\mathbf{x}^{A}\right|}\right]^{4} .
$$

Our ansatz for the homothety is

$$
D^{A i}=\frac{2}{h} x^{A i}
$$

for some constant $h$. It follows from (5.1) and (5.2) that $D$ is a homothety (4.20) if

$$
\left(x^{A i} \partial_{A i}-h\right) L=\frac{h}{2} K
$$

where at this point $K$ can be any function in the kernel of the differential operator of (5.1), i.e.

$$
G_{k l}^{i j} \partial_{A i} \partial_{B j} K=0
$$

We should be careful since $L$ contains a divergent piece that does not contribute to the metric. To see this let us separate out two terms as

$$
L=L_{1}+L_{2}+L^{\prime}
$$

where

$$
L_{1}=-\frac{1}{16 \pi} \sum_{A} m_{A}^{4} \int d^{3} x \frac{1}{\left|\mathbf{x}-\mathbf{x}^{A}\right|^{4}}
$$

and

$$
L_{2}=-\frac{1}{4 \pi} \sum_{A \neq B} m_{A}^{3} m_{B} \int d^{3} x \frac{1}{\left|\mathbf{x}-\mathbf{x}^{A}\right|^{3}\left|\mathbf{x}-\mathbf{x}^{B}\right|}
$$

These are the only two potentially divergent pieces since all other terms in $L$ contain at most an integrable singularity $\left|\mathbf{x}-\mathbf{x}^{A}\right|^{-2}$ as $\mathbf{x} \rightarrow \mathbf{x}^{A}$ (provided that none of the black holes 
are coincident). Moreover, $L^{\prime}$ is homogeneous of degree -1 , i.e. $L^{\prime}\left(\lambda x^{A i}\right)=\lambda^{-1} L^{\prime}\left(x^{A i}\right)$, so Euler's theorem tells us that

$$
\left(x^{A i} \partial_{A i}+1\right) L^{\prime}=0 .
$$

Let us now turn our attention to the divergent terms. First, note that $L_{1}$ is independent of $\mathbf{x}^{A}$ and thus does not contribute to the metric. If we insert a cutoff $\left|\mathbf{x}-\mathbf{x}^{A}\right|>\delta$ in the integral (5.9) we find that

$$
L_{2}=-\sum_{A \neq B} m_{A}^{3} m_{B} \frac{\ln r_{A B}+(1-\ln \delta)}{r_{A B}} .
$$

However, using $G_{k l}^{(i j)}=\delta^{i j} \delta_{k l}$ it is easy to show that

$$
G_{k l}^{i j} \partial_{C i} \partial_{D j} \frac{1}{r_{A B}}=0
$$

so that the $1-\ln \delta$ term does not contribute to the metric. Thus we find that $L_{2}$ is not homogeneous but instead satisfies

$$
\left(x^{A i} \partial_{A i}+1\right) L_{2}=-\frac{1}{2} K
$$

where

$$
K=2 \sum_{A \neq B} m_{A}^{3} m_{B} \frac{1}{r_{A B}}
$$

is in the kernel of $G_{k l}^{i j} \partial_{C i} \partial_{D j}$ by (5.12). So (5.5) holds for $h=-1$ and

$$
D^{A i}=-2 x^{A i}
$$

is a homothetic vector field.

We now show that (4.21) holds, i.e. that

$$
D_{A k}=g_{A k B l} D^{B l}=-2 g_{A k B l} x^{B l}=\partial_{A k} K .
$$

It turns out (as anticipated by the notation) that the solution to this is precisely the function $K$ defined in (5.14). To see this, note that $L$ obeys $L\left(O_{j}^{i} x^{A j}\right)=L\left(x^{A i}\right)$ for any orthogonal matrix $O_{j}^{i}$. Thus

$$
x^{B k} \epsilon_{k}^{m i} \partial_{B i} L=0
$$


since the $\epsilon^{m i}{ }_{k}$ generate the $S O(3)$ symmetry of $\mathbf{R}^{3}$. A straightforward computation then reveals that (5.16) holds. This completes the proof that the bosonic part of the sigma model action admits an $S L(2, \mathbf{R})$ symmetry in the near-horizon limit.

It remains to show that the fermionic terms arising from the supersymmetric completion of the action are also conformally invariant. It is straightforward to verify from (5.15), (5.17) and (3.20)-3.25) that conditions (4.23), (4.26) and 4.27) hold for $\beta=2$. Since we have $h=-1$ the superconformal group is $D(2,1 ; 0)$. This is a semi-direct product of $S U(1,1 \mid 2)$ with $S U(2)$, where the extra $S U(2)$ acts nontrivially on the supercharges of $S U(1,1 \mid 2)$.

The generator $K$ of special conformal transformations has some useful features. Conformal invariance in quantum mechanics was studied in [19,20] (following the more general treatment of [21,22]), wherein it is noted that the hamiltonian $H$ of such a theory possesses neither a ground state nor discrete eigenstates. It was suggested that one should consider, instead of $H$ eigenstates, eigenstates of

$$
L_{0}=\frac{1}{2}(H+K)
$$

which has a well behaved discrete spectrum of normalizable eigenstates. In our case, the near-horizon limit of the black hole moduli space has asymptotically locally flat regions corresponding to near-coincident black holes $r_{A B} \rightarrow 0$ for any $A \neq B$, so the hamiltonian does not have a ground state or discrete eigenstates. However, the function $K$ (5.14) diverges in these noncompact regions of the moduli space. Thus the operator $L_{0}$, in which $K$ serves as a potential to cut off the noncompact regions of the moduli space, will provide a sensible definition of the quantum mechanics, as per the suggestion of [19] (and in a related black hole context of [23,24]). A similar story was recently found for five-dimensional black holes 10,12,25].

\section{Acknowledgements}

It is a pleasure to thank R. Britto-Pacumio, G. Papadopoulos, M. Headrick, R. Jackiw, A. Volovich and especially J. Michelson for many helpful conversations. This work was supported by the NSF graduate fellowship program and DOE grant DE-FG02-91ER40654. 


\section{Appendix A. A Construction of $d=1$ Superconformal Algebras}

A generic superconformal algebra in $d=1$ dimensions contains the conformal group $S L(2, \mathbf{R})$ generated by $H, D$ and $K, \mathcal{N}$ fermionic supercharges $Q^{i}$, an equal number of fermionic partners $S^{i}=i\left[Q^{i}, K\right]$, as well as some number of bosonic $R$-symmetry generators required for closure of the algebra. A classification of the possible $d=1$ superconformal algebras is obtained by reading off from Nahm's classification [26] of superalgebras those in which $S L(2, \mathbf{R})$ is a factored subgroup of the bosonic part of the superalgebra and in which the fermionic generators sit in a spinorial representation of $S L(2, \mathbf{R})$ [27]. The result appears in Table 1.

\begin{tabular}{|c|c|c|c|}
\hline Superalgebra & $\operatorname{dim}(\# b, \# f)$ & $R$-symmetry & Spinor Rep. \\
\hline$O s p(1 \mid 2)$ & $(3,2)$ & 1 & $\mathbf{1}$ \\
\hline$O s p(2 \mid 2)=S U(1,1 \mid 1)$ & $(4,4)$ & $U(1)$ & $\mathbf{2}$ \\
\hline$O s p(3 \mid 2)$ & $(6,6)$ & $S U(2)$ & $\mathbf{3}$ \\
\hline$P S U(1,1 \mid 2)$ & $(6,8)$ & $S U(2)$ & $\mathbf{2} \oplus \overline{\mathbf{2}}$ \\
$D(2,1 ; \alpha)_{0<\alpha \leq 1}$ & $(9,8)$ & $S U(2) \times S U(2)$ & $(\mathbf{2}, \mathbf{2})$ \\
\hline$O s p(5 \mid 2)$ & $(13,10)$ & $S O(5)$ & $\mathbf{5}$ \\
\hline$S U(1,1 \mid 3)$ & $(12,12)$ & $S U(3) \times U(1)$ & $\mathbf{3} \oplus \overline{\mathbf{3}}$ \\
$O s p(6 \mid 2)$ & $(18,12)$ & $S O(6)$ & $\mathbf{6}$ \\
\hline$G(3)$ & $(17,14)$ & $G_{2}$ & $\mathbf{7}$ \\
$O s p(7 \mid 2)$ & $(24,14)$ & $S O(7)$ & $\mathbf{7}$ \\
\hline$O s p\left(4^{*} \mid 4\right)$ & $(16,16)$ & $S U(2) \times S O(5)$ & $(\mathbf{2}, \mathbf{4})$ \\
$S U(1,1 \mid 4)$ & $(19,16)$ & $S U(4) \times U(1)$ & $\mathbf{4} \oplus \overline{\mathbf{4}}$ \\
$F(4)$ & $(24,16)$ & $S O(7)$ & $\mathbf{8}$ \\
$O s p(8 \mid 2)$ & $(31,16)$ & $S O(8)$ & $\mathbf{8}$ \\
\hline$O s p(n \mid 2), n>8$ & $\left(\frac{1}{2} n(n-1)+3,2 n\right)$ & $S O(n)$ & $\mathbf{n}$ \\
$S U(1,1 \mid n), n>4$ & $\left(n^{2}+3,4 n\right)$ & $S U(n) \times U(1)$ & $\mathbf{n} \oplus \overline{\mathbf{n}}$ \\
$O s p\left(4^{*} \mid 2 n\right), n>2$ & $\left(2 n^{2}+n+6,8 n\right)$ & $S U(2) \times S p(2 n)$ & $(\mathbf{2}, \mathbf{2 n})$ \\
\hline \hline
\end{tabular}

Table 1. Lie superalgebras of classical type 3 that contain an $S L(2, \mathbf{R})$ subgroup, adapted from a table in [27]. For clarity we have written out the $\mathcal{N} \leq 8$ algebras explicitly.

We do not differentiate the various real (i.e, noncompact) forms of these algebras in our table. A classification of real simple Lie algebras of classical type appears in [29], and the

3 An excellent resource on Lie superalgebras is [28]. 
results also appear in [27]. The algebra $O s p\left(4^{*} \mid 2 n\right)$ has bosonic part $S O^{*}(4) \times U s p(2 n)$, where $S O^{*}(4) \cong S L(2, \mathbf{R}) \times S U(2)$ is a noncompact form of $S O(4)$. The superalgebra $P S U(1,1 \mid 2)$ is the quotient of $S U(1,1 \mid 2)$, which is not even semi-simple, by the $U(1)$ generated by the identity matrix. It has become common in the physics literature to use $S U(1,1 \mid 2)$ as a shorthand for $P S U(1,1 \mid 2)$, and we adopt this convention throughout this paper.

The Lie superalgebras $D(2,1 ; \alpha)$ with $\alpha \neq 0,-1, \infty$ form a one-parameter family of superalgebras. The algebras with parameters $\alpha, \alpha^{-1}$ and $-1-\alpha$ are isomorphic [28], so it is sufficient to consider the family of algebras $0<\alpha \leq 1$. We have $D(2,1 ; 1)=$ $O \operatorname{sp}(4 \mid 2)=O \operatorname{sp}\left(4^{*} \mid 2\right)$. In the limit $\alpha \rightarrow 0, D(2,1 ; \alpha)$ reduces to a semi-direct product of $S U(1,1 \mid 2)$ with $S U(2)$, with the extra $S U(2)$ acting nontrivially on the fermionic generators of $S U(1,1 \mid 2)$.

We now describe the construction of a general $d=1$ superconformal algebra starting with generators $H, D$ and $K$ satisfying the $S L\left(2, \mathbf{R}\right.$ ) algebra (4.4) and $\mathcal{N}$ supercharges $Q^{i}$ satisfying the supersymmetry algebra

$$
\left\{Q^{i}, Q^{j}\right\}=2 \delta^{i j} H
$$

It follows that

$$
\left[H, Q^{i}\right]=0 .
$$

The first nontrivial constraint is that the $Q^{i}$ must have the appropriate conformal weight,

$$
\left[D, Q^{i}\right]=i Q^{i}
$$

We define the superconformal operators $S^{i}$ through the relation

$$
\left[K, Q^{i}\right]=i S^{i}
$$

Jacobi identities then guarantee that

$$
\left[H, S^{i}\right]=-i Q^{i}, \quad\left[D, S^{i}\right]=-i S^{i},
$$

but one must check that the $S^{i}$ defined by (A.4) satisfyt

$$
\left[K, S^{i}\right]=0 .
$$

4 This is not the only route to take, but it is the one we find most convenient. 
The definition (A.4), together with the appropriate Jacobi identity, fixes the symmetric part of $\left\{Q^{i}, S^{j}\right\}$ to be $\delta^{i j} D$. We then define the $R$-symmetry generator to be the antisymmetric part, so that

$$
\left\{Q^{i}, S^{j}\right\}=\delta^{i j} D+R^{i j}
$$

holds. Note that there are at most $\frac{1}{2} \mathcal{N}(\mathcal{N}-1)$ independent generators $R^{i j}$, so the dimension of the $R$ symmetry algebra of any $d=1$ superconformal algebra with $\mathcal{N}$ supersymmetries can be at most $\frac{1}{2} \mathcal{N}(\mathcal{N}-1)$. This is just a reflection of the fact that the $R$ symmetry algebra for $\mathcal{N}$ real supercharges can be at most $S O(\mathcal{N})$.

Another application of the Jacobi identity gives

$$
\left\{S^{i}, S^{j}\right\}-2 \delta^{i j} K=i\left[K, R^{i j}\right]
$$

The left-hand side is symmetric under $i \leftrightarrow j$ while the right-hand side is antisymmetric, thus both sides must vanish separately. Two more applications of the Jacobi identity give

$$
\left[H, R^{i j}\right]=0=\left[D, R^{i j}\right]
$$

We have found from (A.8) and (A.9) that the $R$ symmetry generators defined by (A.7) commute with $S L(2, \mathbf{R})$.

We have gotten quite far with little effort, but now it is time to pay the piper. As mentioned above, the $\frac{1}{2} \mathcal{N}(\mathcal{N}-1)$ generators $R^{i j}$ may or may not be independent. We therefore rewrite the $R^{i j}$ in terms of $\operatorname{dim}(\mathcal{R})$ independent generators $R^{a}, a=1, \ldots, \operatorname{dim}(\mathcal{R})$. The final constraint on the superalgebra is that the $Q^{i}$ live in a spinor representation of $\mathcal{R}$, i.e.

$$
\left[R^{a}, Q^{i}\right]=i\left(T^{a}\right)^{i}{ }_{j} Q^{j}
$$

where the $\left(T^{a}\right)^{i}{ }_{j}$ satisfy

$$
\left[T^{a}, T^{b}\right]=-f^{a b}{ }_{c} T^{c}
$$

for some constants $f^{a b}{ }_{c}$. The $\left(T^{a}\right)^{i}{ }_{j}$ are the generators of the representation and the $f^{a b}{ }_{c}$ are the structure constants of $\mathcal{R}$. In general, the requirement (A.10) places very strong constraints on the theory in question.

We shall now see that A.10 fixes the rest of the superconformal algebra. Since $K$ commutes with the $R$ symmetry it follows from (A.4) and (A.10) that

$$
\left[R^{a}, S^{i}\right]=i\left(T^{a}\right)^{i}{ }_{j} S^{j}
$$


so the $S^{i}$ necessarily lie in the same representation of $\mathcal{R}$ as the $Q^{i}$. Finally, an application of the Jacobi identity to (A.10) gives

$$
\left[R^{a}, R^{b}\right]=i f^{a b}{ }_{c}^{c}
$$

with the help of (A.11). Note that we have reconstructed the algebra of $\mathcal{R}$ from the representation (A.10), so the representation must be faithful.

This completes the construction of the superconformal algebra. In summary, the construction requires checking (4.4), (A.1), (A.3), (A.6), (A.10), and (A.11).

\section{Appendix B. The $O s p(1 \mid 2)$ Algebra}

The $\mathcal{N}=1$ superconformal algebra $O \operatorname{sp}(1 \mid 2)$ contains $S L(2, \mathbf{R})$ (4.4) as well as two fermionic generators $Q$ and $S$ satisfying

$$
\begin{array}{ccc}
\{Q, Q\}=2 H, & \{S, S\}=2 K, & \{Q, S\}=D, \\
{[H, Q]=0,} & {[D, Q]=i Q,} & {[K, Q]=i S,} \\
{[H, S]=-i Q,} & {[D, S]=-i S,} & {[K, S]=0 .}
\end{array}
$$




\section{References}

[1] R. C. Ferrell and D. M. Eardley, "Slow Motion Scattering And Coalescence Of Maximally Charged Black Holes," Phys. Rev. Lett. 59, 1617 (1987).

[2] G. W. Gibbons and P. J. Ruback, "The Motion Of Extreme Reissner-Nordstrom Black Holes In The Low Velocity Limit," Phys. Rev. Lett. 57, 1492 (1986).

[3] G. W. Gibbons and R. E. Kallosh, "Topology, Entropy And Witten Index Of Dilaton Black Holes," Phys. Rev. D 51, 2839 (1995) arXiv:hep-th/9407118.

[4] G. W. Gibbons, G. Papadopoulos and K. S. Stelle, "HKT and OKT geometries on soliton black hole moduli spaces," Nucl. Phys. B 508, 623 (1997) arXiv:hep-th/9706207.

[5] G. Papadopoulos and A. Teschendorff, "Multi-angle five-brane intersections," Phys. Lett. B 443, 159 (1998) arXiv:hep-th/9806191.

[6] J. Traschen and R. Ferrell, "Quantum mechanical scattering of charged black holes," Phys. Rev. D 45, 2628 (1992) arXiv:hep-th/9205061.

[7] K. Shiraishi, "Moduli space metric for maximally charged dilaton black holes," Nucl. Phys. B 402, 399 (1993).

[8] J. Michelson, "Scattering of four-dimensional black holes," Phys. Rev. D 57, 1092 (1998) arXiv:hep-th/9708091.

[9] J. Michelson and A. Strominger, "The geometry of (super)conformal quantum mechanics," Commun. Math. Phys. 213, 1 (2000) arXiv:hep-th/9907191.

[10] J. Michelson and A. Strominger, "Superconformal multi-black hole quantum mechanics," JHEP 9909, 005 (1999) arXiv:hep-th/9908044.

[11] J. Gutowski and G. Papadopoulos, "The dynamics of very special black holes," Phys. Lett. B 472, 45 (2000) arXiv:hep-th/9910022.

[12] R. Britto-Pacumio, J. Michelson, A. Strominger and A. Volovich, "Lectures on superconformal quantum mechanics and multi-black hole moduli spaces," Proceedings of the NATO ASI on Quantum Geometry, Akureyri, Iceland (1999) arXiv:hep-th/9911066].

[13] J. A. de Azcarraga, J. M. Izquierdo, J. C. Perez Bueno and P. K. Townsend, "Superconformal mechanics and nonlinear realizations," Phys. Rev. D 59, 084015 (1999) |arXiv:hep-th/9810230.

[14] J. Kumar, "Conformal mechanics and the Virasoro algebra," JHEP 9904, 006 (1999) arXiv:hep-th/9901139.

[15] C. M. Hull, "The geometry of supersymmetric quantum mechanics," arXiv:hepth/9910028.

[16] N. Wyllard, "(Super)conformal many-body quantum mechanics with extended supersymmetry," J. Math. Phys. 41, 2826 (2000) arXiv:hep-th/9910160.

[17] G. Papadopoulos, "Conformal and superconformal mechanics," Class. Quant. Grav. 17, 3715 (2000) arXiv:hep-th/0002007. 
[18] R. A. Coles and G. Papadopoulos, "The Geometry Of The One-Dimensional Supersymmetric Nonlinear Sigma Models," Class. Quant. Grav. 7, 427 (1990).

[19] V. de Alfaro, S. Fubini and G. Furlan, "Conformal Invariance In Quantum Mechanics," Nuovo Cim. A 34, 569 (1976).

[20] R. Jackiw, "Dynamical Symmetry Of The Magnetic Monopole," Annals Phys. 129, $183(1980)$.

[21] S. R. Coleman and R. Jackiw, "Why Dilatation Generators Do Not Generate Dilatations?," Annals Phys. 67, 552 (1971).

[22] C. G. Callan, S. R. Coleman and R. Jackiw, "A New Improved Energy - Momentum Tensor," Annals Phys. 59, 42 (1970).

[23] P. Claus, M. Derix, R. Kallosh, J. Kumar, P. K. Townsend and A. Van Proeyen, "Black holes and superconformal mechanics," Phys. Rev. Lett. 81, 4553 (1998) arXiv:hepth/9804177].

[24] G. W. Gibbons and P. K. Townsend, "Black holes and Calogero models," Phys. Lett. B 454, 187 (1999) arXiv:hep-th/9812034.

[25] R. Britto-Pacumio, A. Strominger and A. Volovich, "Two-black-hole bound states," JHEP 0103, 050 (2001) [arXiv:hep-th/0004017.

[26] W. Nahm, "Supersymmetries And Their Representations," Nucl. Phys. B 135, 149 (1978).

[27] P. Claus, R. Kallosh and A. Van Proeyen, "Conformal symmetry on world volumes of branes," Proceedings of the Trieste Conference on Superfivebranes and Physics in $5+1$ dimensions (1998) arXiv:hep-th/9812066.

[28] L. Frappat, P. Sorba and A. Sciarrino, "Dictionary on Lie superalgebras," arXiv:hepth/9607161.

[29] M. Parker, "Classification Of Real Simple Lie Superalgebras Of Classical Type," J. Math. Phys. 21, 689 (1980). 